\title{
VLBI OBSERVATIONS OF GRAVITATIONAL LENS SYSTEMS
}

\author{
Edwin L. Turner \\ Princeton University Observatory \\ Princeton, NJ 08544 USA
}

\begin{abstract}
Four specific and particularly powerful types of possible VLBI lens studies are discussed. First, comparison of mas scale structure in putative pairs of images separated by arc seconds can provide a powerful additional test of the lens hypothesis in specific candidate systems. Second, VLBI searches for lens systems with image separations too small for resolution by optical or VLA searches will limit (or even determine!) the cosmological density of condensed objects with individual masses $\sim 10^{6} \mathrm{M}_{\odot}$. Third, study of multiply imaged superluminal expansion events will allow a determination of the light travel time delay between different images in a lens system, a quantity which is quite difficult to measure by other means but which would allow profound cosmological tests. Fourth, VLBI data can be used to determine relative image parities and even the full magnification matrix of various images in a lens system, thus providing powerful additional constraints on detailed lens models. Finally, the speculative possibility of detecting Galactic stellar lensing events using VLBI techniques is discussed.
\end{abstract}

\section{INTRODUCTION}

Since I have participated only very marginally in VLBI observations of gravitational lens systems and since nearly all of those who have made the major contributions to this fledgling subfield are in attendance and will be reporting many of their results in other papers, no review of the current state of the subject will be given. In addition, although the customary brief summaries of the history and basic ideas of gravitational lens theory were included in the presentation of this paper, they have been stated so well and so often elsewhere (see for example, Canizares 1987, Gott 1987, Turner 1987a, Peacock 1983, Walsh 1983, and Gunn 1981) that they will not be included in the written version. Instead I 
shall here concentrate on the special potential of VLBI observations for helping to realize some of the many possible astrophysical and cosmological applications of gravitational lens studies. In effect this paper is an advertisement intended to promote VLBI observations of gravitational lenses.

Before narrowing the focus to possible VLBI observations, it may be well to briefly state the fundamental scientific justification for studying gravitational lensing. The primary reason for the current level of interest in lenses is that they may provide a fundamentally new tool for attacking many difficult problems in cosmology and extragalactic astronomy. In principle, lens observations could determine all three of the standard cosmological parameters $\left(H_{0}, \Omega_{o}, \Lambda\right)$, allow an independent measurement of galaxy masses, measure or constrain many of the properties of dark matter, facilitate studies of the spatial structure of the IGM, give some indication of the structure of quasars on very small angular scales, help explain apparent clustering on the sky of objects of very different redshifts, account for rare but extreme objects and phenomena (e.g., BL Lacs), and so forth. The list is being steadily expanded by theoretical work. The underlying reasons for this remarkable potential are that lensing involves optical geometry on scales of order the horizon size and that any material which affects the metric, globally or locally, can influence lensing events independent of its emission and absorption properties. It remains to be seen how well many of these potential applications can be achieved in hard observational reality. Nevertheless, these problems are so important and so difficult to address by other available means that enthusiasm for gravitational lens studies is undiminished.

\section{TESTING THE LENSING HYPOTHESIS}

Currently the most vexing observational problem associated with gravitational lens studies is that of deciding whether or not particular observed systems are actually due to lensing (Djorgovski et. al. 1987, Shaver, Wampler, and Cristiani 1987; Turner 1987b). The alternative hypothesis is usually that we are simply observing two or more separate but physically associated (i.e., clustered) quasars with similar properties. The situation is logically difficult since no degree of similarity can absolutely exclude separate twin objects. Oddly, neither can observed differences, particularly small or moderate ones, really exclude the lensing hypothesis since they can quite plausibly be ascribed to source variability plus differential image time delay or to different microlensing structure in the images (Paczynski 1986; Kayser, Refsdal, and Stabell 1986). At present, no clear cut and generally accepted critical test of the lensing hypothesis exists; each case must be judged on an individual basis. 
VLBI observations can provide a very powerful additional test of the lensing hypothesis in systems which are sufficiently strong radio sources by allowing a comparison of the mas scale structure in the separate images. The first order test is just whether or not both images have compact components carrying similar fractions of the total flux. A second order test is possible if high quality VLBI observations yield a phase closure map of the compact components in each image. In this case it should be possible to match these maps to each other after the application of a $2 \times 2$ magnification matrix. This test can only be applied to structures with angular sizes much smaller than the image separation (typically a few arc seconds) otherwise much more complex distortions due to highly variable magnification must be considered. Thus, for currently available observing techniques and known candidate lens systems, it is unique to VLBI. Of course, since many sources show great intrinsic variability in flux and structure on mas scales, differential image time delays can confuse these tests. Comparison of source structure at the same emission epoch may be crucial in some cases (see section 4 below).

VLBI observations of the sort described above have lent important support to the best documented case of gravitational lensing 0957+561 (Gorenstein et. al. 1988, Gorenstein et. al. 1984, Porcas et. al. 1981), have yielded preliminary results for another well studied systems $2016+112$ (Heflin et. al. 1988), and are being pursued for two other lens candidates 0023+171 and 1042+178 (Hewitt 1987).

\section{THE COSMOLOGICAL DENSITY OF LOW MASS COMPACT OBJECTS}

The probability of gravitational lensing events for distant sources is proportional to the mass density of the lensing objects in the intervening volume and, for high redshift sources, is of order the lensing objects' contribution to $\Omega_{o}$ (Turner, Ostriker, and Gott 1984; Press and Gunn 1973). Thus, a determination of the frequency of lensing events (or a limit there on) in a sample of distant objects is of fundamental cosmological significance. In order to detect a lensing event it is generally necessary to resolve two or more of the multiple images of the distant source. For fixed source and lens distances the characteristic image separation is proportional to $M_{\text {lens }}^{1 / 2}$ for compact (i.e., point like) lensing objects. Thus, good angular resolution is necessary to detect lensing events due to low mass objects.

The particular relevance of VLBI observations for this problem arises from the fact that the image separation produced by a lens at a typical redshift $(\sim 0.5)$ for a high redshift $(\sim 2)$ quasar is $Z 2\left(M_{\text {lens }} / 10^{6} M_{\odot}\right)^{1 / 6}$ mas. This means that only VLBI observations can produce a lensing limit on (or detection of!) a 
population of compact objects with masses in the particularly theoretically interesting range near $10^{6} \mathrm{M}_{\odot}$. This mass range corresponds to the Jeans mass in the cosmic plasma just after recombination (Peebles and Dicke 1968) when the development of significant large scale structure in the Universe probably began, agrees with the mass of dark halo objects needed to heat the old Galactic disk population by tidal shocks (Lacey and Ostriker 1985), and is the mass scale for candidate macroscopic forms of dark matter least constrained by other direct and indirect arguments (Carr 1978). Unfortunately the high frequency of intrinsic structure on mas scales in high redshift radio sources renders this otherwise straightforward cosmological test difficult; structure due to lensing must be distinguished from real source structure in some way. Nevertheless, this cosmological experiment is so powerful, so unique, and so important, a serious attempt to carry it out is clearly indicated in my view.

Despite having been first proposed nearly 15 years ago (Press and Gunn 1973), no serious attempt to use VLBI data for the test described above has been carried out to my knowledge. Recently Hewitt (1986) carried out an analogous experiment using VLA data and derived a limit on the cosmic mass density of compact objects with masses near $10^{11} \mathrm{M}_{\odot}$.

\section{DETERMINING DIFFERENTIAL IMAGE TIME DELAYS}

The light travel times along the various paths to the source in a multiple image gravitational lens system are not generally equal both because they are of different physical lengths and because they pass through varying depths of the lens's potential field producing varying gravitational time dilations. Thus the source is seen at slightly different look back times in each image. The differences in these light travel times are differential image time delays $\Delta t$, and we may write $c \Delta t \sim(\Delta \theta)^{2} D_{\text {lens }}$ where $\Delta \theta$ is the image separation (an observable!) and $D_{\text {lens }}$ is the distance to the lens. It should be emphasized that the dimensionless factor required to write this relation as an equality is sensitive to the details of the mass distribution in the lensing object. Thus, a measurement of the differential time delay in a lens systems provides information on a combination of the distance to the lens and its mass distribution. Both are of great interest. A detailed model of the lens is required to realize many of the suggested lens applications, particularly the study of dark matter. If the lens mass distribution can be modeled satisfactorily, the lens distance falls out, and if the lens redshift can be measured, a value of $H_{o}$ follows. This possibility of a direct physical determination of Hubble's constant on a very large scale, a sort of cosmological Holy Grail, is probably the best known and most assiduously pursued potential application of gravitational lenses. 
Since most quasars vary in brightness, the differential image time delays can be measured in principle just by recording the light curves of each image and cross correlating. In practice, a wide variety of intrinsic, technical, practical, and organizational problems make the accumulation of adequate light curve data quite difficult. No unambiguous time delay has yet been determined by this method despite substantial efforts. A possible alternate approach to the problem could be made if VLBI observations of a lens system revealed typical superluminal jet activity. In many superluminal expansion systems two components are observed to be separating with a linear proper motion so that measurement of the separation at any pair of epochs allows extrapolation back to a unique time of zero separation. In the various lens images of a single such superluminal source the zero separation times would differ by the differential time delays which could be determined therefore by a single pair of observational epochs. In reality one would probably want several epochs to check the linearity of the proper motions in each image and to make sure one was viewing the same pair of components in each image and not some previous or successive generation.

Unfortunately, no report of superluminal expansion in a lens system has yet appeared. Continued monitoring of $0957+561$ which has a compact core-jet structure (Porcas et. al. 1981, Gorenstein et. al. 1984) and any other lens system discovered to have suggestive compact structure is clearly warranted.

\section{MEASURING THE MAGNIFICATION MATRIX}

A detailed model of a particular gravitational lens system predicts a mapping of each point in the source plane onto one or more points on the image plane. In any small region of the image plane this mapping can be characterized by a $2 \times 2$ magnification matrix applied to some corresponding region of the source plane (Falco, Gorenstein, and Shapiro 1985). Unfortunately, only a tiny fraction of this rich and complex set of predictions can be tested observationally to determine the validity of the model. Naturally, only those regions of the source plane which are emitting detectably produce any signature in the image plane. Furthermore, if the source cannot be resolved as it appears in the image plane, only ratios of the total area magnification (determinant of the $2 \times 2$ matrix) can be measured. Thus, in typical situations, a variety of detailed lens models can predict degenerate observational consequences.

As mentioned in section 2, VLBI offers the possibility of partially alleviating this problem. Comparison of resolved mas scale structure in lens images separated by arc seconds can lead to a direct determination of the $2 \times 2$ matrix at each image position. Such information can provide powerful and unique constraints on lens models which often must form the basis of other conclusions derived from a particular lens system. 
VLBI studies of the gravitational lens $0957+561$ have achieved this goal and provided crucial inputs to our best detailed models of that system (Falco et. al. 1985).

\section{DETECTING GALACTIC STELLAR GRAVITATIONAL LENSES}

Einstein (1936) published the first detailed analysis of gravitational lensing. He considered lensing of stars in the Galaxy by other stars and, after outlining the basic theory, concluded that no such effect was likely to be observed in practice. His grounds for pessimism were 1) that the multiple images were unlikely to be resolvable, and 2) that such alignments are extremely unlikely. In the next year Zwicky (1937) suggested that extragalactic lensing events would be more observationally accessible, and the subject was thereafter relegated to cosmologists. Here I would like to point out that VLBI observations could contradict these expectations by detecting Galactic stellar lensing events.

First the resolution issue is easily addressed by VLBI. The image separation will be $\Delta \theta \geq 2\left(M / M_{\odot}\right)^{1 / 2}\left(D_{\text {lens }} / 8 \mathrm{kpc}\right)^{-1 / 2}$ mas for an infinitely distant source and half as great for a source at twice the distance of the lens. Thus stars all over the Galaxy will produce detectable image separations at the best achievable VLBI resolutions.

The low probability of lensing events is a more challenging obstacle. A random line of sight through the Galaxy has a probability $\sim 10^{-6}$ of passing within the standard critical impact parameter of a star required to produce a strong lensing event. Of course, we need not choose random lines of sight; directions very close to the Galactic Plane and even toward the Galactic Center may be chosen. This is sufficient to raise the lensing probability to $\sim 10^{-4}$ or even $\sim$ $10^{-3}$ in the most favorable directions. Further improvements are possible if one considers not just strong lensing events but also the more probable large impact parameter cases in which one greatly demagnified and one nearly unperturbed image occur (Hewitt 1988, 1986; Press and Gunn 1973). If VLBI maps with dynamic ranges of order $10^{3}$ are achieved, the chances of detecting an event will be increased by a factor of about 30 so that total probabilities can reach levels of 0.003 up to 0.03 . At this point the detectable lensing frequency produced by Galactic stars is comparable to that due to external galaxies for high redshift quasars (Turner et. al. 1984) and thus not impossibly rare.

What sorts of VLBI sources should be examined for Galactic stellar lensing? Fortuitously positioned extragalactic sources, masers, and perhaps pulsars come to mind. The masers seem most promising to me since they are bright (allowing large dynamic ranges), numerous, concentrated to the Galactic Plane, and merit much observational attention for other reasons in any case. 
How would Galactic stellar lenses be distinguishable from intrinsic source structure? The simplest test would be spectral similarity, in particular identical radial velocities and line shapes. Individual maser components often show complex and individualistic line profiles which should make excellent signatures. A more powerful test could be based on proper motion effects. Galactic rotation will produce relative proper motions of the lens and source of order 6 mas/yr and even parallax effects are not entirely negligible ( $~ Z 0.25$ mas), especially for nearby lensing stars. Of course, the masers themselves also have proper motions. Moreover, since the lensing objects are effectively simple point masses (or at worst, binary systems) and the differential time delays are tiny, there will be little ambiguity in fitting a lens model to observations at a single epoch. Observations at multiple epochs must be consistent with this model and some reasonable relative proper motion of the source and lens. A few epochs of observation should provide an entirely conclusive test of the lens hypothesis.

Finally, other than the minor triumph of proving Einstein wrong (albeit, in a trivial way), what value would observations of Galactic stellar lensing have? The most obvious answer would be that information on stellar masses and distances (and thus on stellar interiors theory and Galactic structure) of a new kind would be forthcoming. One might also learn something about masers (or whatever source), particularly about their very small scale angular structure. A more speculative possibility is that we might learn something of the nature of the "Oort dark matter" which seems to make up roughly half of the material in the Galactic Plane (Bahcall 1984).

\section{CONCLUSIONS}

The two following conclusions suggest themselves: VLBI data will probably prove crucial to achieving many of the most important potential gravitational lens experiments. Gravitational lens observations may well provide some of the most scientifically important applications of VLBI techniques. These logically independent conclusions may be put in a predictive form: Future symposia on gravitational lens will someday feature VLBI observations as prominently as they now feature (for instance) optical spectroscopy, and future symposia on VLBI will eventually devote roughly as much attention to lens results as they now do to (for instance) masers or superluminal sources.

Conversations with numerous colleagues, including particularly B. Burke, M. Gorenstein, J. Hewitt, J. Ostriker, and I. Shapiro, provided the background for this discussion. A. Rogers pointed out the importance of Galactic rotation for the discussion in section 6. This work was supported in part by NASA grant 
NAGW-765 and NSF grant AST84-20352.

\section{REFERENCES}

Bahcall, J. N. 1984, Ap. J., 276, 169.

Canizares, C. 1987, in Observational Cosmology: Proceedings of IAU Symposium No. 124, eds. A. Hewitt and G. Burbidge (Boston: D. Reidel Publishing), in press.

Carr, B. J. 1978, Comm. Ap., 7, 161.

Djorgovski, S., Perley, R., Meylan, G., and McCarthy, P. 1987, preprint.

Einstein, A. 1936, Science, 84, 506.

Falco, E. E., Gorenstein, M. V., and Shapiro, I. I. 1985, Ap. J. Lett., 289, L1.

Gorenstein, M. V., Bonometti, R. J., Cohen, N. L., Falco, E. E., Shapiro, I. I., Bartel, N., Rogers, A. E. E., and Marcaide, J. M. 1988, in this volume.

Gorenstein, M. V., Shapiro, I. I., Rogers, A. E. E., Cohen, N. L., Corey, B. E., Porcas, R. W., Falco, E. E., Bonometti, R. J., Preston, R. A., Rius, A., and Whitney, A. R. 1984, Ap. J., 287, 538.

Gott, J. R. 1987, in Dark Matter in the Universe: Proceedings of IAU Symposium No. 117, eds. J. Kormendy and G. R. Knapp (Boston: D. Reidel Publishing), 219.

Gunn, J. E. 1981, in Proceedings of the Tenth Texas Symposium on Relativistic Astrophysics, eds. R. Ramaty and F. C. Jones (New York: New York Academy of Sciences), 287.

Heflin, M. B., Gorenstein, M. V., Falco, E. E., Shapiro, I. I., Burke, B. F., Hewitt, J. N., Rogers, A. E. E., and Lawrence, C. 1988, in this volume.

Hewitt, J. N. 1986, MIT Ph. D. Thesis Dissertation.

Hewitt, J. N. 1987, private communication.

Hewitt, J. N. 1988, in preparation.

Kayser, R., Refsdal, S., and Stabell, R. 1986, Astron. Ap., 166, 36.

Lacey, C. G., and Ostriker, J. P. 1985, Ap. J., 199, 633.

Paczynski, B. 1986, Ap. J., 301, 76.

Peacock, J. A. 1983, in Quasars and Gravitational Lenses: Proceedings of the 24th Liege International Astrophysical Colloquium, ed. J. P. Swings (Liege: Universite de Liege), 86.

Peebles, P. J. E., and Dicke, R. H. 1968, Ap. J., 154, 891.

Porcas, R. W., Booth, R. S., Browne, I. W. A., Walsh, D., and Wildenson, P. N. 1981, Nature, 289, 758.

Press, W. H., and Gunn, J. E. 1973, Ap. J., 185, 397.

Shaver, P. A., Wampler, E. J., and Cristiani, S. 1987, Nature, $327,40$.

Turner, E. L. 1987a, in Dark Matter in the Universe: Proceedings of IAU Symposium No. 117, eds. J. Kormendy and G. R. Knapp (Boston: D. Reidel Publishing), 227. 
Turner, E. L. 1987b, in Observational Cosmology: Proceedings of IAU Symposium No. 124, eds. A. Hewitt and G. Burbidge (Boston: D. Reidel Publishing), in press.

Turner, E. L., Ostriker, J. P., and Gott, J. R. 1984, Ap. J., 284, 1.

Walsh, D. 1983, in Quasars and Gravitational Lenses: Proceedings of the 24th Liege International Astrophysical Colloquium, ed. J. P. Swings (Liege: Universite de Liege), 106.

Zwicky, F. 1937, Phys. Rev, 51, 290. 